\title{
Efecto de la radiación ultravioleta y la colonización micorrízica en árboles pioneros de la región oriental de América del Norte
}

\section{Effect of ultraviolet radiation and the mycorrhizal colonization in pioneers trees from the eastern region of North America}

\section{Efeito da radiação ultravioleta e colonização micorrízica em árvores pioneiras do leste da América do Norte}

\section{Nilsen Lasso-Rivas}

Biólogo, Magister en Ciencias-Biología, Doctor of Philosophy Major in Ecology and Evolutionary Biology

Programa de Agronomía, Universidad del Pacífico, Kilómetro 13 vía al Aeropuerto Barrio el Triunfo, Buenaventura, Colombia..

nileonard@yahoo.com

\section{Resumen}

Las plantas están expuestas a múltiples factores de estrés que causan alteraciones en su fisiología. Sin embargo, la evidencia sugiere que las relaciones mutualistas con microorganismos pueden ayudar a las plantas a superar los efectos perjudiciales de algunos factores de estrés abiótico. El objetivo de esta investigación fue analizar los efectos individuales e interactivos de los rayos ultravioleta (UV) (280-400 nm) y la simbiosis micorrizíca arbuscular (MA) en el crecimiento vegetativo en plántulas de tres especies de árboles pioneros. Se cultivaron en invernadero plántulas de Populus deltoides, Betula nigra y Salix nigra usando un diseño factorial $2 \times 2$, con dos condiciones de radiación UV (con y sin radiación UV) y dos tratamientos MA (inóculo MA vivo o inóculo MA previamente autoclavado). Después de tres meses, las plantas se cosecharon y se registraron crecimiento, morfología y asignación de biomasa; se evaluó porcentaje de colonización MA mediante técnica de cuadricula. Se encontró que las plántulas ex- puestas a la radiación UV tenían menor peso seco de la raíz, diámetro del tallo, y relación raíz parte aérea $(R / A)$, y mayor área foliar específica (AFE) y razón de área foliar (RAF) que aquellas plantas cultivadas en ausencia de radiación UV. La colonización MA no evidenció aumento en el crecimiento de las plantas. La radiación ultravioleta redujo el porcentaje de raíz colonizada por hongos MA en plántulas de $P$. deltoides y $S$. nigra. Se evidenció que niveles ambientales de radiación UV pueden ejercer un efecto indirecto sobre la micorrización MA en plántulas de árboles pioneros.

Palabras clave: micorrizas arbusculares, estrés abiótico, Populus deltoides, Betula nigra, Salix nigra, crecimiento vegetativo

\section{Abstract}

Plants live in environments where they are exposed to multiple stress factors that cause alterations in their normal physiology. However, the evidence su- 
ggests that mutualistic relationships with microbes can help plants to overcome the detrimental effects of some abiotic stress factors. The goal of this research was to investigate the individual and interactive effects of solar ultraviolet (UV) radiation (280$400 \mathrm{~nm}$ ) and arbuscular mycorrhizal (AM) symbiosis on vegetative growth in seedlings of three shade intolerant species. Seedlings of Populus deltoides, Betula nigra, and Salix nigra were grown in greenhouse under a $2 \times 2$ factorial design, with two UV radiation conditions (with and without UV radiation) and two AM treatments (with normal AM inoculum or with autoclaved inoculum). After three months, plants were harvested and growth, morphology, and allocation variables were measured, percentage of roots colonized by AM fungi was assessed using the grid line intercept technique. The results showed that seedlings exposed to UV radiation had smaller root masses, stem diameters, and root to shoot ratios; and greater specific leaf areas and leaf area ratios. Mycorrhizal colonization did not result in any increase in growth, and in one case the AM plants were smaller than the non-mycorrhizal control. Ultraviolet radiation reduced the percentage of root colonized by AM fungi of $P$. deltoides and $S$. nigra. The results demonstrate that ambient levels of UV radiation can exert an indirect effect on mycorrhizal fungi in the rhizosphere of tree seedlings.

Key-words: arbuscular mycorrhizae, abiotic stress, Populus deltoides, Betula nigra, Salix nigra, seedling growth, greenhouse experiment.

\section{Resumo}

As plantas estão expostas a múltiplas pressões que causam alterações na sua fisiologia. No en- tanto, as evidências sugerem que as relações mútuas com microorganismos pode ajudar as plantas a superar os efeitos nocivos de alguns fatores de estresse abióticos. O objetivo desta pesquisa foi analisar os efeitos individuais e interactivos de radiação ultravioleta (UV) (280-400 $n m$ ) e simbiose micorrízicos arbusculares (MA) sobre o crescimento vegetativo em mudas de três espécies de árvores pioneiras. Foram cultivadas em casa de vegetação, as plântulas de Populus deltoides, Betula nigra e Salix nigra, utilizando um delineamento experimental fatorial $2 \times 2$, com duas condições de radiação UV (com e sem radiação UV) e dois tratamentos MA (inóculo MA vivo ou inóculo MA previamente autoclavado). Depois de três meses, as plantas foram colhidas e registrou-se o crescimento, morfologia e alocação de biomassa. Foi avaliada, a percentagem de coIonização dá MA, pela técnica de grade. Verificouse que as plântulas expostas à radiação UV apresentaram menor peso seco da raiz, diâmetro do caule, raízes e parte aérea relação $(R / A)$, e maior área foliar específica (SLA) e razão de área foliar (RAF), que aquelas plantas cultivadas na ausência de radiação UV. A colonização MA não mostrou nenhum aumento no crescimento das plantas. A radiação ultravioleta reduziu a porcentagem de fungos de raízes colonizadas por MA em plântulas de $P$. deltóides e $S$. nigra. Foi demonstrado que os níveis ambientais de radiação UV podem ter um efeito indireto sobre a micorrização MA em plântulas de árvores pioneiras.

Palavras-chave: fungos micorrízicos arbusculares, estresse abiótico, Populus deltoides, Betula nigra, Salix nigra, crescimento vegetativo

\section{Introducción}

Las plantas viven expuestas a múltiples factores de estrés que pueden alterar su fisiología normal (Alexieva et al., 2003). Es por esto que las plantas han desarrollado un conjunto de mecanismos que les permiten detectar y responder a los diferentes factores de estrés, reduciendo al mínimo sus efectos negativos (Atkinson \& Urwin, 2012). Además de los mecanismos de protección contra el estrés, las relaciones mutualistas con microorganismos de la rizosfera, como bacterias y hongos simbióticos, pueden ayudar a las plantas superar los efectos perjudiciales de algunos factores 
de estrés abiótico (Grover et al., 2010). Dado que es muy probable que debido al cambio climático en el futuro se dé un aumento en el número y la intensidad de eventos ambientales extremos ( $\mathrm{Li}-$ chtenthaler 1996, Alexieva et al., 2001, Dukhovskis et al., 2003), es importante estudiar el efecto de los factores de estrés abiótico en las interacciones simbióticas en las plantas.

La radiación ultravioleta (UV) es un factor de estrés abiótico importante y generalizado para las plantas (Singh et al., 2006). El espectro electromagnético de la radiación UV se divide para fines prácticos en tres bandas de frecuencia: radiación UV-C (200-280 nm), radiación UV-B (280-315 nm) y radiación UV-A (315-400 $\mathrm{nm}$ ). La radiación UV-C es la más energética, pero es completamente absorbida por la atmósfera superior y no llega a la superficie de la Tierra. Tanto la radiación UV-B como la radiación UV-A penetran en la troposfera, sin embargo, la mayor parte de la radiación UV-B es absorbida por el ozono estratosférico (Frederick, 1993). El estudio de los efectos de la radiación UV en los ecosistemas se vio estimulado debido a la preocupación por la disminución en la capa de ozono causada por la descarga antropogénica de los clorofluorocarbonos (CFC) y el consiguiente aumento de la radiación UV-B a nivel del suelo (Frederick, 1993). Por esa razón, la comprensión de los efectos ecofisiológicos de la radiación ultravioleta en los ecosistemas se ha centrado en la irradiancia UV-B por encima de los niveles ambientales actuales (Phoenix et al., 2003). Sin embargo, la intensidad de la radiación UV que llega a la superficie de la tierra se ve afectada no sólo por la columna de ozono, sino también por otros factores como el ángulo solar, la elevación, la nubosidad, y la contaminación de la troposfera por materiales particulados (Pablo \& Gwynn-Jones, 2003). Así, futuras variaciones en los niveles de radiación UV resultado de cambios en el clima pueden tener mayor impacto en los ecosistemas terrestres que los cambios debidos al agotamiento del ozono estratosférico (Ballare et al. 2011). Los resultados de las investigaciones sobre los efectos del aumento de la radiación UV-B en plántulas muestran cambios en la morfología de éstas como lo son reducción de la altura, diámetro, biomasa y área foliar. Estas respuestas se pueden atribuir a: (i) cambios en el metabolismo de hormonas, específicamente auxinas, que están implicadas en diferentes procesos de desarrollo (Huang et al., 1997; Jansen, 2002), (ii) cambio en la asignación de carbono hacia la producción en las hojas de compuestos fotoprotectores tales como flavonoides y ácidos fenólicos (Schumaker et al., 1997; Warren et al., 2003; Kotilainen et al., 2008; Morales et al., 2010.); y (iii) cambios en la expansión de las hojas como resultado del aumento en la actividad de las peroxidasas de la pared celular (Tegelberg et al., 2001; Wargent et al., 2009;. Robson \& Aphalo, 2012). Igualmente, la radiación UV-B puede dañar directamente el complejo proteico del fotosistema II, lo que resulta en una reducción de la tasa de fotosíntesis neta (Hideg et al., 1993). Menos conocidos son los efectos de la radiación UV-A, que también provoca efectos biológicos significativos.

Los estudios de suplementación de radiación UV-B mediante lámparas han sido muy útiles para develar los efectos de este tipo de radiación en las plantas, estos estudios debe ser complementados con experimentos de exclusión de radiación UV mediante filtros de plástico. Los estudios de exclusión permiten la identificación de los efectos de los niveles ambientales de UV en las plantas (Sullivan 2005; Amudha et al., 2005). Por otra parte, existe una creciente evidencia de que la radiación UV-A también provoca respuestas en las plantas que a veces son opuestas a aquellas provocadas por la radiación UV-B, un fenómeno que podría ser el resultado de los diferentes fotorreceptores que median las respuestas las radiaciones UV-B y UV-A (Ryel et al., 2010). Por lo tanto, es de esperar que la exclusión de los dos tipos de radiación ultravioleta produzca resultados diferentes a los de la exclusión de un solo tipo. Por ejemplo, Kotilainen et al. (2008) informan de un experimento en campo con árboles de Alnus incana y Betula pubescens en el que la concentración de compuestos fenólicos en las hojas variaba de manera diferente en respuesta a la exclusión de la radiación UV-B, o de la exclusión de la radiación UV-A/UV-BV. 
La simbiosis micorrízica es probablemente la asociación mutualista más extendida entre plantas y hongos con más de $90 \%$ de todas las especies de plantas presentando algún tipo de asociación con hongos micorrízicos (Aerts, 2002). La simbiosis micorrízica arbuscular (HMA) es el tipo más común; en la simbiosis HMA los hongos penetran en las células corticales y forman grupos de hifas finamente divididos conocido como arbúsculos, también forman vesículas dentro y fuera de las células corticales (Turk et al., 2006). Los resultados de múltiples investigaciones indican que los hongos micorrízicos pueden ayudar a las plantas a hacer frente a diferentes tipos de estrés, esto a través de la promoción del crecimiento de la planta y a la resistencia inducida a enfermedades (Pineda et al., 2010). Por ejemplo, las micorrizas pueden estimular el crecimiento de plantas mediante la mejora de la absorción de nutrientes, especialmente fósforo (Smith et al., 2011). Del mismo modo, las micorrizas pueden mejorar la capacidad de absorción de agua de las plantas al aumentar la conductividad hidráulica de la raíz, mejorando así la capacidad de las plantas para resistir la sequía (Turk et al 2006; Ruiz-Lozano 2003). Además, los hongos micorrízicos pueden proporcionar protección contra ciertas enfermedades de la raíz (Jeffries et al., 2003).

Es interesante que muchos estudios muestran que la radiación UV-B puede afectar de manera negativa la colonización de raíces por parte de HMA (Klironomos, 1995; Van de Staaij et al., 2001; Zaller et al., 2002). Este efecto indirecto de la radiación UV sobre la colonización HMA se puede atribuir a cambios inducidos por la radiación UV en el equilibrio de fitohormonas en la planta huésped, ya que la evidencia indica que la colonización de micorrizas puede ser en parte regulada desde el lado de la planta por la acción de fitohormonas (Hause et al., 2007). Por ejemplo, el ácido abscísico (ABA) contribuye a la susceptibilidad del tomate a la infección por HMA y parece jugar un papel importante en el desarrollo de los arbúsculos (Herrera-Medina et al., 2007). Del mismo modo, auxinas tales como el ácido indolacético (IAA) pueden estimular la colonización HMA de las raíces al incrementar el número de raíces finas durante las fases tempranas de crecimiento (Hause et al., 2007). Los estudios mencionados anteriormente han dado alguna información sobre el efecto de la radiación UV-B en la simbiosis micorrízica. Sin embargo, todavía hay un conocimiento limitado sobre el efecto que tienen los niveles ambientales de la radiación UV sobre la simbiosis micorrízica.

El objetivo de esta investigación fue analizar los efectos individuales e interactivos de la radiación UV y la colonización HMA en el crecimiento vegetativo de plántulas de Populus deltoides, Salix nigra, y Betula nigra, tres especies de árboles que no toleran crecer bajo la sombra. Estas especies fueron elegidas porque son de rápido crecimiento, están adaptados a altos niveles de radiación UV, y pueden tener una mayor respuesta a las micorrizas que las especies tolerantes a la sombra (Siqueira et al., 1998; Zangaro et al., 2005). Se plantearon cuatro hipótesis: (1) la exclusión de radiación UV alterará el crecimiento, la morfología y la distribución de biomasa en plántulas de $P$. deltoides, B. nigra, y S. nigra, (2) la colonización HMA tendrá un efecto positivo en el crecimiento de las plántulas, (3) la exclusión de la radiación UV aumentará el porcentaje de colonización de raíces por HMA, y (4) habrá una interacción significativa entre la radiación UV y la colonización de micorrizas, de forma tal que la colonización MA evitara, al menos en parte, los efectos perjudiciales de la radiación UV.

\section{Materiales y Métodos}

\section{Material vegetal}

Las especies de árboles evaluadas en este estudio se encuentran comúnmente en hábitats abiertos a lo largo de las riberas de los ríos en la parte oriental de los Estados Unidos (Jurgensen et al., 1996). Populus deltoides Bartram. ex Marshall (Salicácea) es una especie pionera que ha sido objeto de intensa investigación para su uso en las industrias de la 
madera y la pulpa de papel, y como posible cultivo energético forestal (Bradshaw et al. 2000). Salix nigra Marshall (Salicáceas) es una especie pionera común en los márgenes de los ríos y pantanos, es la única de cerca de 90 especies de sauces nativos de América del Norte con importancia comercial (Burns \& Honkala, 1990). Betula nigra L. (Betulaceae) es una especie de crecimiento rápido que crece en las tierras bajas a lo largo de las riberas y zonas húmedas en el este de América del Norte (Coyle \& Bolita, 1982).

\section{Diseño experimental}

Los experimentos se llevaron a cabo en los invernaderos de la Universidad Estatal de lowa, en Ames, lowa, EE.UU. Se recogieron semillas de $P$. deltoides, $S$. nigra y $B$. nigra en diferentes localidades en Ames (42 02 '05 "N $93^{\circ} 37$ '12" W, 287 $\mathrm{m}$ de altitud). Veinte (20) semillas de cada especie se sembraron en macetas en forma de cono (Ray Leasch Cono-contenedores: volumen 0,15 L, $205 \mathrm{~mm}$ de longitud, $40 \mathrm{~mm}$ de diámetro superior; Stuewe and Sons, Corvallis, Oregon, EE.UU.) que contenían una mezcla 1:1 de arena y suelo, con un pH 6.7 (en agua) previamente esterilizada en un autoclave durante 90 min. Dos semanas después de la germinación se ralearon todos los contenedores y se dejó una sola plántula con un tamaño que oscilaba de 1,5 a $1,7 \mathrm{~cm}$. El diseño experimental consistió en un modelo factorial, con dos niveles de radiación UV (con y sin radiación UV) y dos condiciones MA (inoculo MA esterilizado e inoculo MA vivo).

\section{Condiciones de crecimiento}

Debido a la atenuación parcial de la radiación UV producida por el vidrio del invernadero se hizo necesario el uso de radiación UV suplementaria proporcionada por lámparas fluorescentes. La radiación suplementaria se suministró diariamente por un período de 10 horas utilizando lámparas fluorescentes UV-A340Q-Panel (emiten radiación de $365 \mathrm{~nm}$ a $295 \mathrm{~nm}$ con un pico de emisión en 340nm, Q-Panel, Cleveland, Ohio EE.UU.). Las lámparas estaban suspendidas $10 \mathrm{~cm}$ por encima de marcos de PVC de 6 m ' 0,9 m, los cuales estaban cubiertos con plástico de diacetato de celulosa, este plástico permite el paso de la radiación UV-A/B. Para el tratamiento de exclusión de radiación UV (-UV), los contenedores se colocaron al interior de marcos de PVC similares a los arriba descritos pero en lugar de diacetato de celulosa se usó poliéster el cual no permite el paso de la radiación UV-A/B. La radiación UV-A/B se registró con un medidor de radiación ultravioleta Solarmeter ${ }^{\circledR}$, modelo 5.7 UVA + B Sensitive microvatio Version (Solartech, Inc., Harrison Township, Michigan, EE.UU.), a la altura del dosel a mediodía cada dos semanas durante el transcurso de cada experimento. La densidad de flujo de fotones fotosintéticos (PPFD, 400-700nm) se tomó a mediodía al interior de los marcos de PVC con un cuantómetro Field Scout Quantum Meter® (Spectrum Technologies Inc., de Plainfield, Illinois, EE.UU.). Para minimizar los efectos de la variación microambiental, las posiciones de las plantas dentro de los marcos de PVC y dentro de cada invernadero se rotaron a diario.

\section{Inoculación micorrízica}

La infección micorrízica fue establecida mediante la adición de $6 \mathrm{~g}$ de SYMBIVIT® endomicorriza (Symbiom, Lanskroun, República Checa), este inóculo contiene una mezcla de fragmentos raíces colonizadas, micelios y esporas de Glomus mosseae, G. microagregatum, G. claroideum, G. intraradices, $G$. entunicatum, y $G$. geosporum. Estas especies de HMA usualmente son compatibles con las especies de plantas evaluadas. Las plántulas del control recibieron la misma cantidad de SYMBIVIT® previamente esterilizado en autoclave (121 ${ }^{\circ} \mathrm{C}$ por $\left.30 \mathrm{~min}\right)$.

\section{Análisis de crecimiento}

El número de hojas y la altura de las plantas se midieron cada15 días. Las plantas se cosecharon después de tres meses, en el momento de la cosecha se midió el diámetro del tallo por encima 
del cuello de la raíz, cada plántula fue dividida en hojas, tallo y raíces. Las hojas fueron escaneadas y el área foliar se determinó utilizando el software Compu Eye, Leaf \& software Area ${ }^{\circ}$ (Bakr, 2005).
Las muestras se secaron a $70^{\circ} \mathrm{C}$ durante 48 horas, luego se pesaron y se calcularon diferentes parámetros de crecimiento para cada una de las plantas (Tabla 1).

Tabla 1. Parámetros de crecimiento, morfología, y asignación de biomasa evaluados, abreviaturas utilizadas y las unidades en que estos se expresan.

\begin{tabular}{lll}
\hline Abreviatura & \multicolumn{1}{c}{ Significado } & \multicolumn{1}{c}{ Unidades } \\
\hline $\mathrm{A}$ & Altura & $\mathrm{cm}$ \\
$\mathrm{NH}$ & Número de hojas & Sin unidades \\
$\mathrm{AF}$ & Área foliar & $\mathrm{cm}^{2}$ \\
DT & Diámetro del tallo & $\mathrm{mm}$ \\
PSH & Peso seco de hojas & $\mathrm{G}$ \\
PSA & Peso seco aéreo & $\mathrm{G}$ \\
PSR & Peso seco de raíz & $\mathrm{G}$ \\
PST & Peso seco total & $\mathrm{G}$ \\
R/A & Relación peso seco aéreo/raíz (PSR / PSA) & $\mathrm{g} \cdot \mathrm{g}^{-1}$ \\
AFE & Área foliar especifica (AF/PSH) & $\mathrm{cm}^{2} \cdot \mathrm{g}^{-1}$ \\
RAF & Relación de área foliar (AF/PST) & $\mathrm{cm}^{2} \cdot \mathrm{g}^{-1}$ \\
\hline
\end{tabular}

Determinación del porcentaje de colonización de las raíces

Durante la cosecha se tomó una muestra de la raíz (1-2 g) de cada plántula para hacer la cuantificación de la colonización micorrizica. Las muestras de raíces se aclararon en una solución de $\mathrm{KOH}$ al $10 \%$, se acidificaron en $\mathrm{HCl}$ al $1 \%$, y se tiñeron con azul de tripano $(0,05 \%)$ en glicerol ácido (Robertson et al., 1999). Posteriormente, las muestras se almacenaron en una solución 1: 1: 1 de agua, glicerina y ácido láctico, hasta que fueron examinadas. En el momento del examen cada muestra se esparció en la base de una caja de Petri $(8,5$ $\mathrm{cm}$ diámetro) que tenía una cuadrícula inscrita, y se observó con un microscopio de disección. Cada intersección entre una línea de la cuadricula y una raíz se clasificó como micorrízica (en caso de haber presencia de estructuras fúngicas) o no micorrízica, todas las intersecciones fueron registradas. A continuación, la muestra fue redistribuida y el proceso se repitió dos veces para tener un total de tres conteos. El porcentaje de coIonización HMA se calculó como la relación entre el número de intersecciones con presencia de estructura HMA y el número total de intersecciones multiplicado por 100 (Brundrett et al., 1996).

\section{Análisis estadístico}

El programa R-2.15.1 se utilizó para realizar el análisis estadístico, el nivel de significancia se fijó en 0,05 . Con el fin de reducir el número de pruebas realizadas dado el gran número de variables registradas y puesto que los tamaños de las muestras eran pequeños en relación con el número de variables, se utilizó un análisis de varianza multivariado permutacional (PERMANOVA) para identifi- 
car los efectos significativos de la exclusión de la radiación UV, el inóculo MA, y sus interacciones. Se realizaron pruebas de ANOVA permutacional (Manly, 2007) para ayudar a determinar las variables que contribuyeron a las diferencias significativas observadas en el análisis multivariado. Se empleó la prueba HSD de Tukey para probar las diferencias entre las medias. Los porcentajes de colonización de la raíz se transformaron para lograr la normalidad y cumplir con los supuestos de los análisis estadísticos paramétricos. Las variables de crecimiento, morfología y asignación de biomasa, evaluadas se describen en la Tabla 1. Igualmente, se realizó un meta-análisis utilizando el software MetaWin 2 (Rosenberg et al., 1999), con el objetivo de identificar los efectos globales de la radiación UV y la colonización MA en los tres experimentos.

\section{Resultados}

\section{Condiciones ambientales}

Los experimentos con $P$. deltoides y $S$. nigra se realizaron entre marzo y mayo de 2013. Durante ese período de tiempo la temperatura media del aire fue de $25,6 \pm 0,5^{\circ} \mathrm{C}\left(\min .17,5^{\circ} \mathrm{C}\right.$, máx. $41^{\circ}$ C) y la humedad relativa promedio fue $51,2 \pm 1,7 \%$ (min. 21\%, máx. 91\%). La PPFD medida al interior del marco de PVC cubierto con diacetato de celulosa a mediodía y bajo condiciones de cielo despejado fue en promedio $1325 \pm 78 \mathrm{m \mu} \mathrm{mol} \mathrm{m} \mathrm{m}^{-2}$ $\mathrm{s}^{-1}$ (min. $649 \mathrm{m \mu} \mathrm{mol} \mathrm{m}{ }^{-2} \mathrm{~s}^{-1}$, máx. $1741 \mathrm{m \mu} \mathrm{mol}$ $\left.\mathrm{m}^{-2} \mathrm{~s}-1\right)$. La radiación UV-A/B al mediodía fue en promedio $15 \pm 0,7 \mathrm{~W} \mathrm{~m}^{-2}$ (min. $6 \mathrm{~W} \mathrm{~m}-^{2}$, máx. $32 \mathrm{~W}$ $\mathrm{m}^{-2}$ ). La PPFD al interior del marco de PVC cubierto con poliéster fue $7 \%$ mayor que la registrada al interior del marco cubierto de celulosa, el promedio fue de $1438 \pm 117 \mathrm{m \mu} \mathrm{mol} \mathrm{m}{ }^{-2} \mathrm{~s}^{-1}(\min .585$ $\mathrm{m} \mu \mathrm{mol} \mathrm{m} \mathrm{m}^{-2} \mathrm{~s}^{-1}$, máx. $1900 \mathrm{~m} \mu \mathrm{mol} \mathrm{m}^{-2} \mathrm{~s}^{-1}$ ) y la irradiancia UV-A/B fue de $0 \mathrm{~W} \mathrm{~m}^{-2}$. El experimento con $B$. nigra se realizó entre abril y junio de 2013. Durante el experimento, la temperatura media del aire fue de $26,2^{\circ} \mathrm{C} \pm 0,4^{\circ} \mathrm{C}\left(\min .17,4^{\circ} \mathrm{C}\right.$, máx. $41,8^{\circ} \mathrm{C}$ ) y la humedad relativa promedio fue de 52 $\pm 0,6 \%$ (min. 21\%, max. 91\%). La PPFD medida al interior de la cubierta de diacetato de celulosa fue de $1346 \pm 60 \mathrm{m \mu} \mathrm{mol} \mathrm{m}{ }^{-2} \mathrm{~s}^{-1}\left(\min .649 \mathrm{~m} \mu \mathrm{mol} \mathrm{m}^{-2}\right.$ $\mathrm{s}^{-1}$, máximo $1.800 \mathrm{~mol} \mathrm{~m}^{-2} \mathrm{~s}^{-1}$.) y la radiación UV-A / B promedio fue de $15 \pm 0,6 \mathrm{~W} \mathrm{~m}^{-2}\left(\min .7 \mathrm{~W} \mathrm{~m}^{-2}\right.$, máx. $32 \mathrm{~W} \mathrm{~m}-2)$. La PPFD al interior de la película de poliéster fue de $1447 \mathrm{mu} \mathrm{mol} \mathrm{m}^{-2} \mathrm{~s}^{-1} \pm 80$, (min. $649 \mathrm{mu} \mathrm{mol} \mathrm{m}{ }^{-2} \mathrm{~s}^{-1}$, máx. $1.998 \mathrm{~mol} \mathrm{~m}^{-2} \mathrm{~s}^{-1}$ ) esto es un $7 \%$ más alto que la registrada en el interior del marco recubierto con diacetato de celulosa; la radiación $\mathrm{UV}-\mathrm{A} / \mathrm{B}$ fue de $0 \mathrm{~W} \mathrm{~m}^{-2}$.

\section{Mediciones de crecimiento}

Los resultados del PERMANOVA para cada uno de los tres experimentos mostraron que el conjunto de variables dependientes (Tabla 1) no se vio afectado significativamente por el inóculo MA. Sin embargo, fueron significativamente afectados por la radiación UV $\left(P\right.$. deltoides $F_{2,60}$ $=23.72, \mathrm{P} \leq 0,01$; $S$. nigra $F_{2,50}=14.81, \mathrm{P} \leq 0,01$; y $B$. nigra $\left.F_{2,60}=9 \cdot 51, \mathrm{P} \leq 0,01\right)$. En comparación con las plántulas cultivadas en presencia de radiación UV, las plántulas de $P$. deltoides que crecieron en su ausencia tuvieron menor área foliar, área foliar específica (AFE), y razón de área foliar (RAF) (Figura 1, Tabla 2). El AFE es una medida del grosor de la hoja y / o la densidad del tejido foliar (bajo SLA indica hojas gruesas o densas); la RAF es una medida de la superficie fotosintética con relación a la masa total de la planta. Los análisis de varianza no detectaron efectos interactivos entre la radiación UV y el inoculo MA para ninguna de las variables en ninguna de los tres especies (Tabla 2). Las plántulas de $S$. nigra, cultivadas sin radiación UV tuvieron tallos más largos y gruesos, y mayor peso seco de raíces y peso seco total que aquellas plántulas cultivadas en presencia de la radiación UV (Figura 1, Tabla 2). La exclusión de la radiación UV también resulto en mayor AFE y RAF (Figura 1, Tabla 2). En comparación con las plántulas cultivadas con radiación UV, las plántulas de $B$. nigra cultivados en el tratamiento de exclusión UV tenían significativamente menor AFE y RAF (Figura 1, Tabla 2). 


\section{Colonización de las raíces}

Todas las plántulas de $P$. deltoides, $B$. nigra y $S$. nigra inoculadas con inoculo vivo presentaron estructuras MA al interior de las raíces, aquellas inoculadas con inóculo esterilizado no presentaron evidencia de colonización MA. Los resultados del ANOVA mostraron que, para las plántulas de $P$. deltoides, el inóculo MA resultó en tallos más cortos y de menor diámetro de ( $\mathrm{P}<0,004$, Tabla 2). No hubo correlación significativa entre el porcentaje de colonización y la altura $(R=-0,27, n=30, p=0,15)$. Curiosamente, hubo diferencia significativa en el peso seco total entre plántulas de $P$. deltoides inoculadas y no inoculadas cuando crecieron sin radiación UV, pero no hubo diferencias en el peso seco total entre las dos condiciones MA cuando las plántulas crecieron expuestas a la radiación UV (Figura 1).
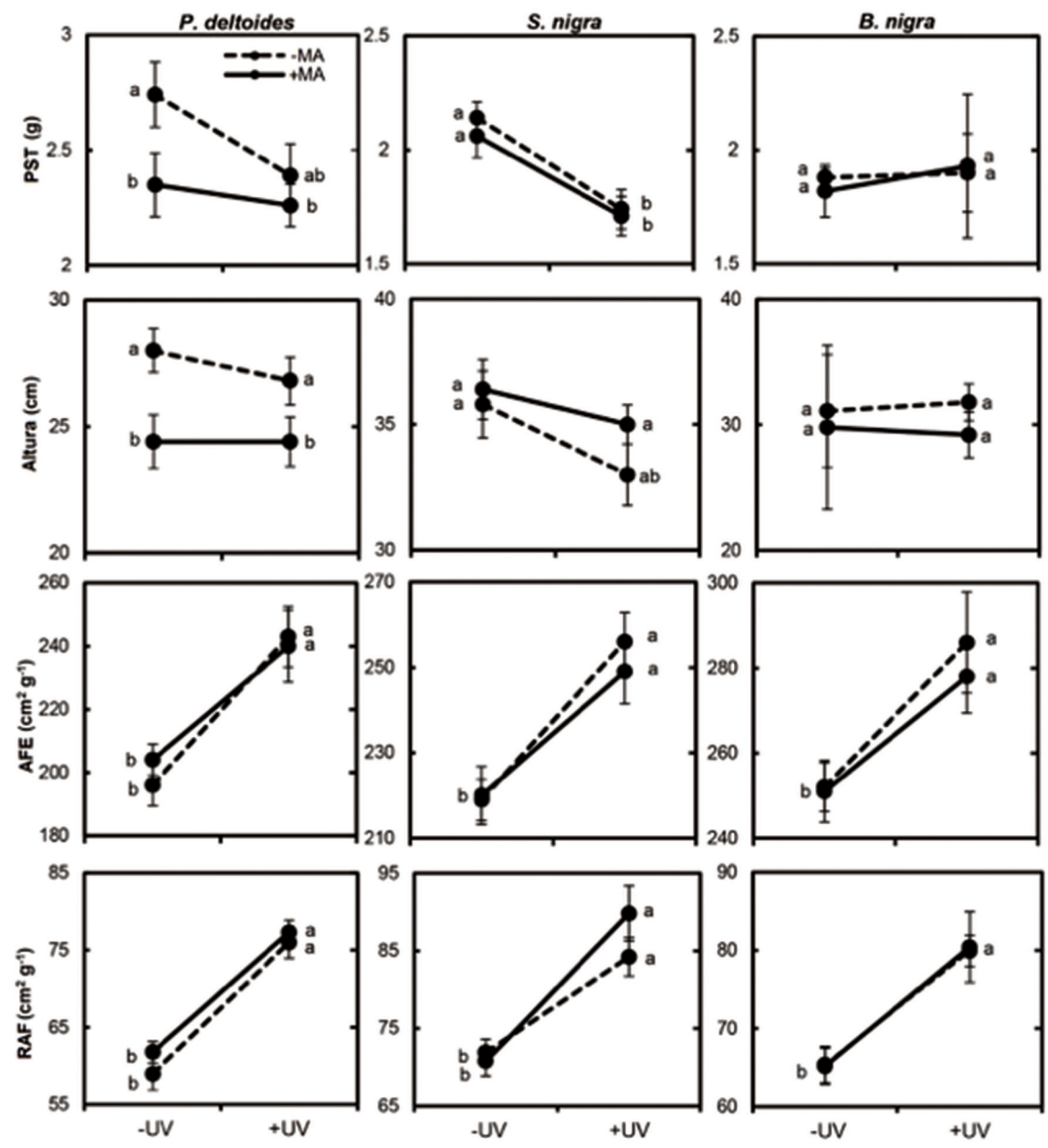

Figura 1. Efectos de la radiación ultravioleta y la colonización micorrízica en la altura, PST, AFE y RAF de $P$. deltoides, $B$. nigra y $S$. nigra plántulas. Cada valor es la media \pm ES. Medias con letras diferentes son significativamente diferentes ( $P<0,004$, test post-ANOVA Tukey).

Los resultados de ANOVA (Tabla 2) mostraron que, para las plántulas de $S$. nigra, la inoculación MA no tuvo efectos significativos sobre las variables asociadas a la morfología o al crecimiento, pero si lo fue para la variable asociada a asignación de biomasa: la relación raíz parte aérea $(R / A)$.
La relación $\mathrm{R} / \mathrm{A}$ en plántulas colonizadas fue de 0,7 mientras que para las plántulas no colonizadas esta proporción fue de 0,78. En el caso de las plántulas de $B$. nigra, no hubo diferencias significativas entre las dos condiciones MA para ninguna de las variables evaluadas (Tabla 2). 
Tabla 2. Resumen de los análisis estadísticos de los efectos de la radiación UV y la colonización micorrízica.

\begin{tabular}{|c|c|c|c|c|}
\hline Especie & Parámetro & UV (1) & HMA (1) & UV x HMA (1) \\
\hline \multirow[t]{5}{*}{ P. deltoides } & altura $(\mathrm{cm})$ & 0.34 & 10.19 ** & 0.34 \\
\hline & Diámetro del tallo $(\mathrm{cm})$ & 7.67 & $10.43^{\star}$ & 2.1 \\
\hline & Área foliar $\left(\mathrm{cm}^{2}\right)$ & $11.65^{\star *}$ & 2.53 & 0.11 \\
\hline & $\operatorname{AFE}\left(\mathrm{cm}^{2} \cdot \mathrm{g}^{-1}\right)$ & $24.69^{\star * \star}$ & 0.15 & 0.47 \\
\hline & $\operatorname{RAF}\left(\mathrm{cm}^{2} \cdot \mathrm{g}^{-1}\right)$ & $80.19^{\star \star \star}$ & 1.69 & 0.16 \\
\hline \multirow[t]{7}{*}{ S. nigra } & Diámetro del tallo $(\mathrm{cm})$ & $13.74^{\star \star \star}$ & 0.13 & 0.2 \\
\hline & Peso seco aéreo (g) & $14.2^{* * *}$ & 0.34 & 0.64 \\
\hline & Peso seco de raíz (g) & $20.72^{\star \star \star}$ & 4.74 & 0.22 \\
\hline & Peso seco total (g) & $20.12^{\star \star \star}$ & 0.39 & 0.09 \\
\hline & $\operatorname{AFE}\left(\mathrm{cm}^{2} \cdot \mathrm{g}^{-1}\right)$ & $26.10^{\star * \star}$ & 0.23 & 0.34 \\
\hline & $\operatorname{RAF}\left(\mathrm{cm}^{2} \cdot \mathrm{g}^{-1}\right)$ & $40.05^{\star \star \star}$ & 0.76 & 1.83 \\
\hline & $\mathrm{T}: \mathrm{R}$ & 0.14 & $12.48^{\star \star \star}$ & 3.98 \\
\hline \multirow[t]{2}{*}{ B. nigra } & $\operatorname{AFE}\left(\mathrm{cm}^{2} \cdot \mathrm{g}^{-1}\right)$ & $11.31^{*}$ & 0.81 & 0.16 \\
\hline & $\operatorname{RAF}\left(\mathrm{cm}^{2} \cdot \mathrm{g}^{-1}\right)$ & $30.57^{* * *}$ & 0.6 & 0.01 \\
\hline
\end{tabular}

Los valores estadísticos tabulados son los valores de $F$ de las pruebas de ANOVA para el efecto principal de la radiación UV (UV), la colonización micorrizica (HMA), y la interacción entre ellos (UV x HMA), los grados de libertad están entre paréntesis. Niveles de significancia con corrección de Bonferroni para análisis de varianza $\left({ }^{* * *} p<0,0001 ;{ }^{* \star} p<0,001\right.$; $\left.{ }^{*} P<0,005\right)$.

Solo se muestran aquellos variables con diferencias significativas a $P<0.004$.

\section{Efecto de la radiación UV sobre la colonización micorrízica}

Las plántulas de $P$. deltoides y $S$. nigra que crecieron en ausencia de radiación UV tuvieron una mayor extensión en la colonización micorrízica que aquellas que crecieron en presencia de radiación UV (Tabla 3). El porcentaje promedio de colonización para plántulas de $P$. deltoides cultivadas bajo exclusión de radiación UV fue de $40 \%$ frente al $30 \%$ para las cultivadas en presencia de radiación UV (Figura 2). Por otro lado, las plántulas de S. nigra cultivadas en condiciones de exclusión UV tuvieron en promedio una colonización HMA de $11 \%$, frente al 4,3\% para las plántulas cultivadas en presencia de radiación UV (Figura 2). En plántulas de $B$. nigra la colonización HMA no fue significativamente afectada por la radiación UV (Figura 2).

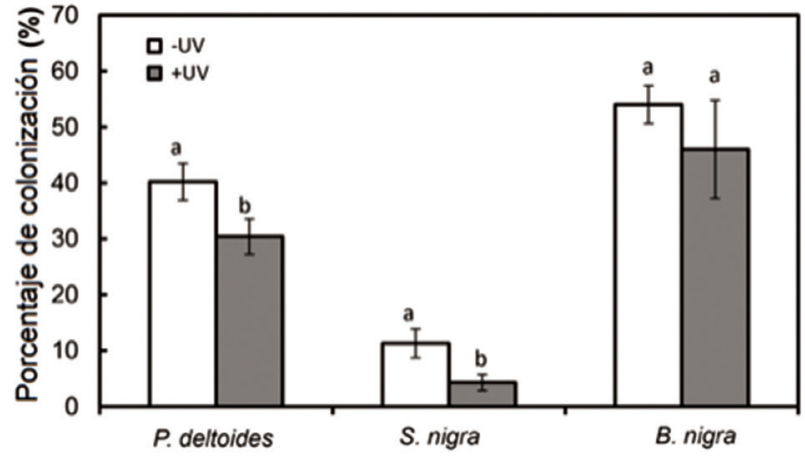

Figura 2. Efecto de la radiación UV sobre la porcentaje de colonización HMA en plántulas de $P$. deltoides, $S$. nigra y $B$. nigra. Las barras representan la media $\pm E S$; barras con letras diferentes son significativamente diferentes ( $P<0.05$, prueba post-ANOVA Tukey). 
Tabla 3. Efecto de la radiación UV sobre la colonización micorrízica en plántulas de $P$. deltoides, $B$. nigra y $S$. nigra.

\begin{tabular}{llll}
\hline Especie & $\boldsymbol{F}$ & gl & $\mathbf{N}$ \\
\hline P. deltoides & $4.56^{*}$ & 1 & 30 \\
S. nigra & $5.95^{\star}$ & 1 & 25 \\
B. nigra & $0.86^{\mathrm{ns}}$ & 1 & 10 \\
\hline
\end{tabular}

Valores de $F$ y niveles de significación para ANOVA $\left({ }^{*} p<0,05 ;{ }^{* *} p<0,01 ;{ }^{* *} p<0,001\right)$.

\section{Efectos generales}

En un meta-análisis se examinan los resultados de diferentes experimentos para probar si juntos demuestran un efecto que es grande, moderado, pequeño, o no diferente de cero (Gurevitch et al. 1992). El método meta-analítico que se usó en esta investigación se basa en la estimación de la magnitud del efecto de interés de las variables en cada uno de los tres experimentos. Los resultados de los meta-análisis mostraron que en general, la radiación UV tiene un efecto negativo en el diámetro del tallo, peso seco de la raíz, y en la relación $\mathrm{R} / \mathrm{A}$; y tiene un efecto positivo en el AFE y RAF (Figura 3). En el caso de la colonización MA, el efecto de esta sobre las variables evaluadas no fue significativamente diferente de cero excepto para el peso seco total, que fue menor en las plántulas colonizadas (Figura 4). Del mismo modo, los resultados de los meta-análisis mostraron que la radiación UV redujo la tasa de colonización MA.

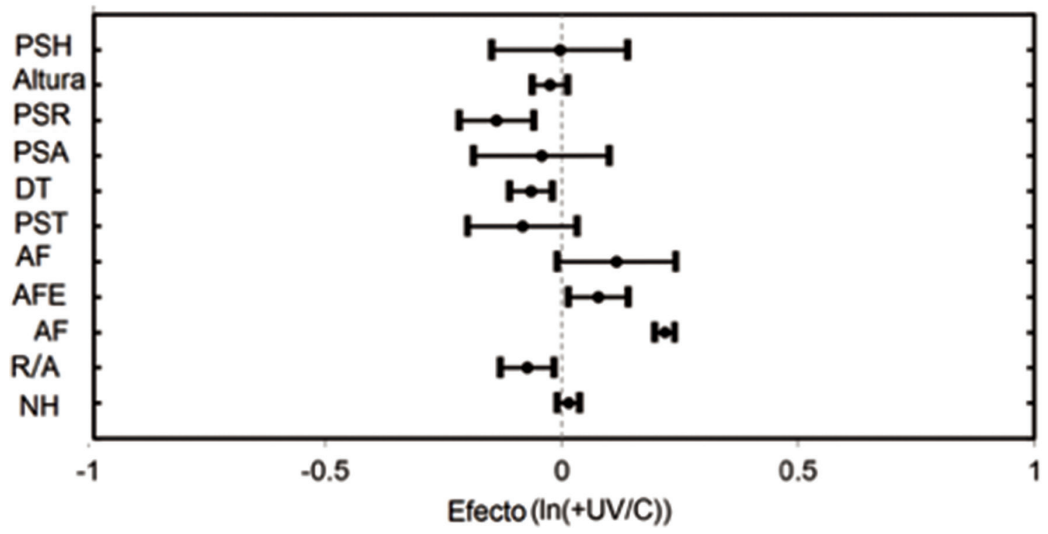

Figura 3. Efecto de la radiación UV sobre los parámetros de crecimiento en las especies evaluadas. Cada línea representa un intervalo de confianza (IC) del 95\% para el tamaño del efecto de las variables de cada una de las tres especies; el efecto de la radiación UV se considera neutral para las variables cuyo $\mathrm{Cl}$ contienen al cero.

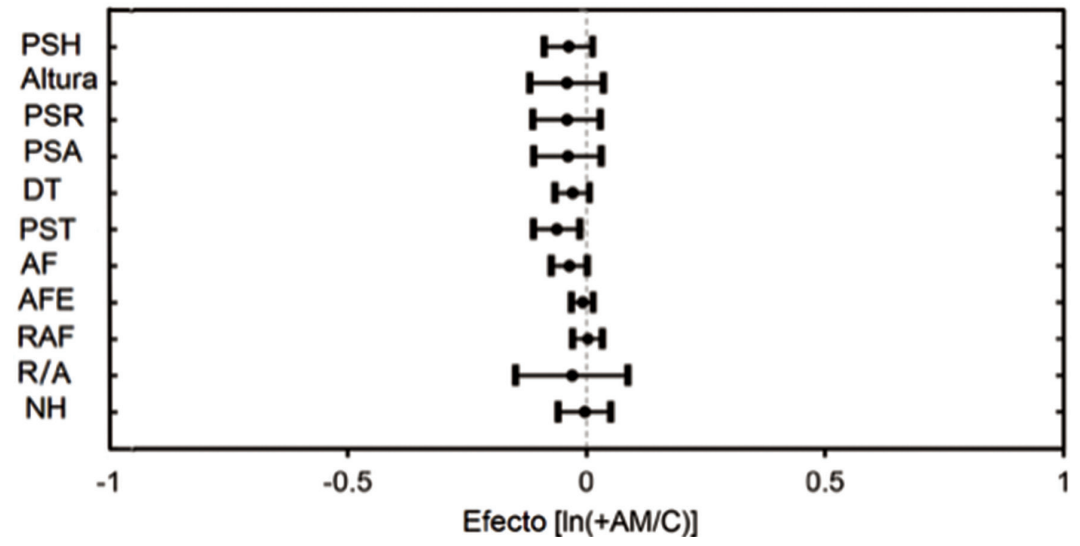

Figura 4. Efecto de AM colonización en los parámetros de crecimiento a travésde las especies evaluadas. Cada línea representa un intervalo de confianza del 95\% (IC)para el tamaño del efecto de las variables de cada una de las tres especies; el efectode la colonización AM se considera neutral para aquellas variables cuyo IC contiene al cero. 


\section{Discusión}

\section{Efecto de la radiación UV}

La exclusión de la radiación UV produjo un aumento de la biomasa en una de las tres especies evaluadas, y produjo menores valores para las variables AFE y RAF en las tres especies (Figura 1 Tabla 2). Estos resultados proporcionan apoyo parcial para la hipótesis (1) de que la exclusión de la radiación UV podría afectar el crecimiento, morfología y distribución de biomasa en plántulas de $P$. deltoides, $B$. nigra, y $S$. nigra. Los resultados muestran que la radiación UV indujo de forma consistente cambios en la morfología de las hojas. Es generalmente aceptado que la radiación UV es un factor de estrés que puede inducir respuestas morfogenéticas en hojas tales como: cambios en el espesor de la hoja y alargamiento (Weih et al., 1998; Bassman et al., 2001; Ren et al., 2006; Yang \& Yao 2.008; Xu et al., 2010). El tipo de alteraciones en AFE y RAF inducidas por la exclusión de la radiación UV que aquí se registran son similares a los reportados por Schumaker et al. (1997); ellos encontraron un aumento en el grosor de hojas en esquejes de Populus trichocarpa cuando las plantas fueron cultivadas en un ambiente con niveles reducidos de radiación UV-B. El aumento en el grosor de la hoja se explicó como el resultado de mucho más parénquima en empalizada, que a su vez dio lugar a un aumento en la tasa de fotosíntesis en comparación con esquejes cultivados en niveles normales de radiación UV-B. En la presente investigación, solo las plántulas de $S$. nigra tuvieron menor peso seco total cuando crecieron en presencia de radiación UV. Puesto que una reducción en la acumulación de biomasa se considera un indicador fiable de la sensibilidad de las plantas a la radiación UV ya que representa la acumulación de los efectos deletéreos de ésta sobre la fisiología de las plantas (Smith, 2000), la reducción en el peso seco total indica que las plántulas de $S$. nigra pueden ser más sensibles a los efectos de la radiación UV que las plántulas de $P$. deltoides y $B$. nigra. Este resultado sugiere que la radiación UV podría ser un factor que influye de manera importante en la interacción entre estas especies.

\section{Efecto del inóculo MA}

Aunque se observaron estructuras MA en las raíces de todas las plántulas expuestas al inoculo vivo, la colonización micorrízica no resulto en un mayor crecimiento de las plantas, y en un caso las plantas colonizadas fueron en promedio más pequeñas que las del control (Figura 1). Este resultado no es compatible con la hipótesis (2) de que la colonización micorrizíca tendría un efecto positivo en el crecimiento de las plántulas. Aunque las micorrizas se consideran generalmente una asociación mutualista en la cual ambas especies se benefician, en condiciones particulares de alta o baja disponibilidad de fósforo la colonización por HMA puede dar lugar a un falta de respuesta de crecimiento o incluso a una respuesta negativa en el crecimiento (Smith et al., 2009). Por lo tanto, algunos autores señalan que las respuestas de las plantas a la colonización micorrízica pueden variar en un continuo que va desde la mutualista hasta la parasitaria, apareciendo la relación parasitaria cuando los costos de la colonización, en términos de carbono, superan los beneficios de una mayor absorción de fósforo (Johnson et al., 1997; Schroeder \& Janos, 2004). Los resultados aquí presentados sugieren una relación de tipo comensalista, de hecho, una observación común en las tres especies fue la alta frecuencia de aparición de vesículas y muy pocos arbúsculos. Mientras que las vesículas son consideradas como estructuras especializadas en la acumulación de productos de almacenamiento, los arbúsculos se consideran el sitio de transferencia de fosfato por parte del hongo a la planta (Fitter, 2006). Las hifas intercelulares se consideran los sitios donde se realiza la transferencia recíproca de carbono de la planta al hongo (Smith et al., 2001). Los resultados también indican efectos diferenciales de la colonización micorrízica sobre las especies evaluadas. Es bien conocido que la colonización por HMA puede dar lugar a diferentes efectos sobre distintas especies de plantas e incluso a nivel intraespecífico (Munkvold et al., 2004). 


\section{Efecto interactivo}

El efecto positivo de la exclusión de la radiación UV en el porcentaje de colonización micorrízica en plántulas de $P$. deltoides y $S$. nigra apoyan la hipótesis (3) de que la exclusión de radiación UV se traduciría en un aumento en la tasa de colonización HMA. Este resultado es consistente con otros estudios; por ejemplo, van de Staaij et al. (2001), en un experimento de campo con Calamagrostis epigeios y Carex arenaria expuestas a altos niveles de radiación UV-B, reportaron la reducción de la tasa de colonización MA. El mayor porcentaje de colonización micorrízica en plántulas cultivadas en ausencia de radiación UV reportado en este estudio, se puede explicar cómo el resultado de más recursos disponibles para la formación de micorrizas. Puesto que la respuesta típica de las plantas a la radiación UV es la producción de compuestos fotoprotectores, es de esperar que en ausencia de radiación UV se dé una reducción en la concentración de tales compuestos. Por ejemplo, en un estudio de campo con plantas de Populus trichocarpa, Schumaker et al. (1997) encontraron una reducción en la concentración de flavonoides en las hojas de las plantas que crecieron expuestas a bajos niveles de radiación UV-B. Una reducción en la producción de compuestos fotoprotectores implica la posibilidad de que más recursos queden disponibles para ser asignados a la formación de micorrizas. Sin embargo, contrario a la hipótesis (4), no hubo interacciones entre el inoculo MA y la radiación UV para ninguna de las variables evaluadas; todos los efectos observados fueron claramente atribuibles ya sea a la radiación UV o al inoculo MA.

\section{Efectos generales (meta-análisis)}

Los efectos generales de la radiación UV en las plántulas incluyen la reducción en el diámetro del tallo, peso seco de la raíz, y la relación R/A (Figura 3). Del mismo modo, las plántulas que crecieron expuestas a la radiación UV tuvieron menor AFE y RAF (Figura 3). Por otra parte, aunque las especies mostraron marcadas diferencias en sus porcentajes de colonización micorrízica (Figura 2), el efecto global de la colonización micorrízica fue una reducción en la acumulación de biomasa (Figura 4). Los resultados muestran que los niveles ambientales de la radiación UV pueden ejercer un efecto indirecto sobre las micorrizas en las plántulas de las especies evaluadas.

\section{Agradecimientos}

Esta investigación se realizó en cumplimiento de un requisito parcial para optar por el título de PhD. en el Departamento de Ecología, Evolución y Biología de Organismos de lowa State University. El autor desea agradecer al Dr. James Raich por sus asesorías y revisión del manuscrito; a los doctores Dean Adams y Dianne Cook por la asistencia con los análisis estadísticos. También se desea agradecer a los doctores Thomas Jurik, Brian Wilsey y Richard Hall por su ayuda en la revisión del manuscrito. El autor está en deuda con Paul Frater por su ayuda con el trabajo con las micorrizas. Igualmente, el autor expresa agradecimiento al Department of Ecology Evolution and Organismal Biology de lowa State University.

\section{Literatura citada}

1. Aerts, R. (2002). The role of various types of mycorrhizal fungi in nutrient cycling and plant competition. In: Heijden MGA, Sanders IR (eds) Mycorrhizal Ecol. Springer Berlin Heidelberg, Berlin, Heidelberg, pp 117-134

2. Alexieva, V., Sergiev, I., Mapelli, S. \& Karanov, E. (2001). The effect of drought and ultraviolet radiation on growth and stress markers in pea and wheat. Plant, Cell Environ 24:1337-1344.

3. Amudha, P., Jayakumar, M. \& Kulandaivelu, G. (2005). Impacts of Ambient Solar UV (280-400 nm) Radiation on Three Tropical Legumes. J Plant Biol 48:284-291.

4. Atkinson, N.J. \& Urwin, P.E. (2012). The interaction of plant biotic and abiotic stresses: from genes to the field. $J$ Exp Bot 63:3523-43.

5. Bakr, E.M. (2005). A new software for measuring leaf area, and area damaged by Tetranychus urticae Koch. J Appl Entomol 129:173-175.

6. Ballaré, C.L., Caldwell, M.M., Flint, S.D. et al. (2011). Effects of solar ultraviolet radiation on terrestrial ecosystems. Patterns, mechanisms, and interactions with climate change. Photochem Photobiol Sci 10:226-41. 
7. Bassman, J.H., Robberecht, R. \& Edwards, G. (2001). Effects of Enhanced UV B Radiation on Growth and Gas Exchange in Populus deltoides Bartr . ex Marsh Author (s): John H . Bassman , Ronald Robberecht, Gerald E . Edwards Published by: The University of Chicago Press Stable. 162:103-110. Recuperado de: http://www.jstor.org/

8. Bradshaw, H.D., Ceulemans, R., Davis, J. \& Stettler, R. (2000). Emerging Model Systems in Plant Biology: Poplar (Populus) as A Model Forest Tree. J Plant Growth Regul 19:306-313.

9. Brundrett, M., Bougher, N., Dell, B. et al. (1996). Working with Mycorrhizas in Forestry and Agriculture. ACIAR Monograph 32, Canberra

10. Burns, R.M. \& Honkala, B.H. (1990). Silvics of North America: Volume 2. Hardwoods. United States Department of Agriculture, Forest Service

11. Coyle, B.F., Sharik, T.L. \& Ferrer, P. (1982). Variation in leaf morphology among disjunct and continuous populations of river birch (Betula nigra L.). Silvae Genet 31:122-125.

12. Dukhovskis, P., Juknys, R., Brazaityte, A. \& Zukauskaite, I. (2003). Plant Response to Integrated Impact of Natural and Anthropogenic Stress Factors. Russ J Plant Physiol 50:147-154.

13. Fitter, A.H. (2006). What is the link between carbon and phosphorus fluxes in arbuscular mycorrhizas? A null hypothesis for symbiotic function. New Phytol 172:3-6.

14. Frederick, J.E. (1993). Ultraviolet sunlight reaching the earth's surface: a review of recent research. Photochem Photobiol 57:175-178.

15. Grover, M., Ali, S.Z., Sandhya, V. et al. (2010) Role of microorganisms in adaptation of agriculture crops to abiotic stresses. World J Microbiol Biotechnol 27:1231-1240.

16. Gurevitch, J., Morrow, L.L., Wallace, A. et al. (1992) A Meta-Analysis of Competition in Field Experiments. Am Nat 140:539-572.

17. Hanlon, M.T. \& Coenen, C. (2011). Genetic evidence for auxin involvement in arbuscular mycorrhiza initiation. New Phytol 189:701-9.

18. Hause, B., Mrosk, C., Isayenkov, S. \& Strack, D. (2007). Jasmonates in arbuscular mycorrhizal interactions. Phytochemistry 68:101-10.

19. Herrera-Medina, M.J., Steinkellner, S., Vierheilig, H. et al. (2007) Abscisic acid determines arbuscule development and functionality in the tomato arbuscular mycorrhiza. New Phytol 175:554-64.

20. Hideg, É., Sass, L., Barbato, R. \& Vass, I. (1993). Inactivation of photosynthetic oxygen evolution by UV-B irradiation: A thermoluminescence study. Photosynth Res 38:455-462.

21. Huang, S., Dai, Q., Peng, S. et al. (1997). Influence of supplemental ultraviolet-B on indoleacetic acid and calmodulin in the leaves of rice (Oryza sativa L.). Plant Growth Regul 21:59-64.

22. Jansen, M.K. (2002). Ultraviolet-B radiation effects on plants: induction of morphogenic responses. Physiol Plant 116:423-429.
23. Jeffries, P., Gianinazzi, S., Perotto, S. et al. (2003). The contribution of arbuscular mycorrhizal fungi in sustainable maintenance of plant health and soil fertility. Biol Fertil Soils 37:1-16.

24. Johnson, N.C., Graham, J.H. \& Smith, F.A. (1997). Functioning of mycorrhizal associations along the mutualism-parasitism continuum. New Phytol 135:575585.

25. Jurgensen, M.F., Richter, D.L., Davis, M.M. et al. (1996). Mycorrhizal relationships in bottomland hardwood forests of the southern United States. Wetl Ecol Manag 4:223-233.

26. Klironomos, J.N. \& Allen, M.F. (1995). UV-B-mediated changes on below-ground communities associated with the roots of Acer saccharum. Funct Ecol 9:923-930.

27. Kotilainen, T., Tegelberg, R., Julkunen-Tiitto, R. et al. (2008). Metabolite specific effects of solar UV-A and UV-B on alder and birch leaf phenolics. Glob Chang Biol 14:1294-1304.

28. Lichtenthaler, H.K. (1996). Vegetation Stress: an Introduction to the Stress Concept in Plants. J Plant Physiol 148:4-14.

29. Manly, B.F.J. (2007). Randomization, Bootstrap and Monte Carlo Methods in Biology, 3rd ed. London

30. Morales, L.O., Tegelberg, R., Brosché, M. et al. (2010). Effects of solar UV-A and UV-B radiation on gene expression and phenolic accumulation in Betula pendula leaves. Tree Physiol 30:923-34.

31. Munkvold, L., Kjøller, R., Vestberg, M. et al. (2004). High functional diversity within species of arbuscular mycorrhizal fungi. New Phytol 164:357-364.

32. Paul, N.D. \& Gwynn-Jones, D. (2003). Ecological roles of solar UV radiation : towards an integrated approach. Trends Ecol Evol 18:48-55.

33. Phoenix, G.K., Gwynn-Jones, D., Lee, J.A. \& Callaghan, T.V. (2003). Ecological importance of ambient solar ultraviolet radiation to a sub-arctic heath community. Plant Ecol 165:163-174.

34. Pineda, A., Zheng, S.-J., van Loon, J.J.A. et al. (2010). Helping plants to deal with insects: the role of beneficial soil-borne microbes. Trends Plant Sci 15:507-514.

35. Pozo, M.J., Jung, S.C. \& Lòpez-Ràez, J. A-AC. (2007). Impact of arbuscular mycorrhizal symbiosis on plant response to biotic stress: the role of plant defence mechanisms. In: Koltai H KY (ed) Arbuscular mycorrhizas Physiol. Funct. Springer Berlin Heidelberg, pp 193-207

36. Pühler, A., Strack, D., Hause, B. et al. (2007). Jasmonates in arbuscular mycorrhizal interactions. Phytochemistry 68:101-110.

37. Ren, J., Yao, Y., Yang, Y. et al. (2006). Growth and physiological responses to supplemental UV-B radiation of two contrasting poplar species. Tree Physiol 26:665-72.

38. Robertson, G.P., Coleman, D.C., Bledsoe, C.S. \& Sollins, P. (1999). Standard soil methods for long-term ecological research. Oxford University Press

39. Robson, T.M. \& Aphalo, P.J. (2012). Species-specific effect of UV-B radiation on the temporal pattern of leaf growth. Physiol Plant 144:146-60. 
40. Rosenberg, M.S., Adams, D.C. \& Gurevitch, J. (1999). MetaWin 2.0 (User's Manual): Statistical Software for Meta-Analysis. 128.

41. Ruiz-Lozano, J.M. (2003). Arbuscular mycorrhizal symbiosis and alleviation of osmotic stress. New perspectives for molecular studies. Mycorrhiza 13:309-17.

42. Ryel, R.J., Flint, S.D. \& Barnes, P.W. (2010). Solar UV-B Radiation and Global Dimming : Effects on Plant Growth and UV-Shielding. In: Wei Gao, Slusser JR, Schmoldt DL (eds) UV Radiat. Glob. Clim. Chang. Springer Berlin Heidelberg, pp 370-394

43. Schroeder, M.S. \& Janos, D.P. (2004). Phosphorus and intraspecific density alter plant responses to arbuscular mycorrhizas. Plant Soil 264:335-348.

44. Schumaker, M.A., Bassman, J.H., Robberecht, R. \& Radamaker, G.K. (1997). Growth, leaf anatomy, and physiology of Populus clones in response to solar ultraviolet-B radiation. Tree Physiol 17:617-26.

45. Singh, S.S., Kumar, P. \& Ashwani, K.R. (2006). Ultraviolet radiation stress: molecular and physiological adaptations in trees. Abiotic Stress Toler. plants. Springer Netherlands, pp 91-110

46. Siqueira, O., Aurelio, M., Rosado, S. \& Davide, C. (1998). Mycorrhizal colonization and mycotrophic growth of native woody species as related to successional groups in Southeastern Brazil. 241-252.

47. Smith., F.A., Grace, E.J. \& Smith, S.E. (2009). More than a carbon economy: nutrient trade and ecological sustainability in facultative arbuscular mycorrhizal symbioses. New Phytol 182:347-58.

48. Smith, J. (2000). Shoot Dry Weight, Chlorophyll and UV-B-absorbing Compounds as Indicators of a Plant's Sensitivity to UV-B Radiation. Ann Bot 86:1057-1063.

49. Smith, S.E., Dickson, S. \& Smith, F.A. (2001). Nutrient transfer in arbuscular mycorrhizas: how are fungal and plant processes integrated? Funct Plant Biol 28:685-696.

50. Smith, S.E., Jakobsen, I., Grønlund, M. \& Smith, F.A. (2011). Roles of arbuscular mycorrhizas in plant phosphorus nutrition: interactions between pathways of phosphorus uptake in arbuscular mycorrhizal roots have important implications for understanding and manipulating plant phosphorus acquisition. Plant Physiol 156:1050-7.

51. van de Staaij, Rozema, J. van Beem, A. \& Aerts, R. (2001). Increased solar UV-B radiation may reduce infection by arbuscular mycorrhizal fungi (AMF) in dune grassland plants: evidence from five years of field exposure. Plant Ecol 154:169-177.
52. Sullivan, J.H. (2005). Possible impacts of changes in UV-B radiation on North American trees and forests. Environ Pollut 137:380-389.

53. Tegelberg, R., Julkunen-Tiitto, R. \& Aphalo, P.J. (2001). The effects of long-term elevated UV-B on the growth and phenolics of field-grown silver birch (Betula pendula). Glob Chang Biol 7:839-848.

54. Turk, M.A., Assaf, T.A. \& Hameed, K.M. (2006). Significance of mycorrhizae. World J Agric Sci 2:16-20.

55. Wargent, J.J., Moore, J.P., Roland Ennos, A. \& Paul, N.D. (2009). Ultraviolet radiation as a limiting factor in leaf expansion and development. Photochem Photobiol 85:279-86.

56. Warren, J.M., Bassman, J.H., Fellman, J.K. et al. (2003). Ultraviolet-B radiation alters phenolic salicylate and flavonoid composition of Populus trichocarpa leaves. Tree Physiol 23:527-35.

57. Weih, M., Johanson, U. \& Gwynn-Jones, D. (1998). Growth and nitrogen utilization in seedlings of mountain birch (Betula pubescens ssp. tortuosa) as affected by ultraviolet radiation (UV-A and UV-B) under laboratory and outdoor conditions. Trees 12:201-207.

58. Xu, X., Zhao, H., Zhang, X. et al. (2010). Different growth sensitivity to enhanced UV-B radiation between male and female Populus cathayana. Tree Physiol 30:148998.

59. Yang, Y.Q. \& Yao, Y. (2008). Photosynthetic responses to solar UV-A and UV-B radiation in low-and high-altitude populations of Hippophae rhamnoides. Photosynthetica 46:307-311.

60. Zaller, J.G., Caldwell, M.M., Flint, D.S. et al. (2002). Solar UV-B radiation affects below-ground parameters in a fen ecosystem in Tierra del Fuego, Argentina: implications of stratospheric ozone depletion. Glob Chang Biol 8:867-871.

61. Zangaro, W., Nishidate, F.R., Camargo, F.R.S. et al. (2005). Relationships among arbuscular mycorrhizas, root morphology and seedling growth of tropical native woody species in southern Brazil. J Trop Ecol 21:529-540.

\section{Conflicto de Intereses}

El autor declara no tener ningún conflicto de intereses 